\title{
Revisión de estudios sobre comunicación para la salud sexual y reproductiva en México, entre 1994 y 2018
}

\section{Review of studies on communication for sexual and reproductive health in Mexico, between 1994 and 2018}

\author{
Julio César Aguila Sáncheza , Karla Y. Covarrubias Cuéllarb, Marina Vázquez Guerreroc \\ a Doctorado en Ciencias Sociales, Universidad de Colima, México \\ b Centro Universitario de Investigaciones Sociales, Universidad de Colima, México \\ ${ }^{\mathrm{C}}$ Escuela de Mercadotecnia, Universidad de Colima, México
}

\section{Resumen}

Introducción: los estados del arte son investigaciones bibliográficas que permiten encontrar antecedentes de un objeto de estudio; ello implica hacer un balance de los principales avances y retrocesos de su estado de la cuestión, con base en los aportes, tendencias y regularidades halladas. Objetivo: presentar un estado del arte sobre la comunicación para la salud sexual y reproductiva destinada a adolescentes y jóvenes de México. Metodología: realizamos una revisión de tipo narrativa, con el fin de evaluar, interpretar y sintetizar las características y hallazgos de los textos analizados. Ello permitió sistematizar los conocimientos que se han generado sobre la problemática en México, haciendo énfasis en la manera en que se ha comprendido y tratado la temática, sus concepciones, modelos, discursos, enfoques, propuestas, aciertos y limitaciones. Resultados: son privilegiados los estudios acerca de la relación comunicación-institución, la efectividad de la comunicación, la evaluación de los componentes del proceso comunicativo, así como las teorías y discursos de la educación sexual en México. Conclusión: los estudios revisados demuestran la importancia de superar las teorías del comportamiento, para desentrañar los condicionamientos estructurales, que subyacen en las problemáticas de salud sexual y reproductiva en adolescentes y jóvenes.

Palabras clave: comunicación para la salud; salud sexual y reproductiva; estado del arte; México.

\begin{abstract}
Introduction: the states of art are bibliographic investigations that allow finding precedents of an object of study. This implies taking stock of the main advances and setbacks in its state of affairs, based on the contributions, trends and regularities found. Objective: to present a state of the art on communication for sexual and reproductive health for adolescents and youth in Mexico. Methodology: we conducted a study of narrative review, in order to evaluate, interpret and synthesize the characteristics and findings of the analyzed texts. This allowed systematizing the knowledge that has been generated about the problem in Mexico, emphasizing the way in which the subject has been understood and treated, its conceptions, models, speeches, approaches, proposals, successes and limitations. Results: the studies on the communication-institution relationship, the effectiveness of communication, the evaluation of the components of the communicative process, as well as the theories and discourses of sexual education in Mexico are privileged. Conclusion: The studies reviewed demonstrate the importance of overcoming behavioral theories, in order to unravel the structural conditions that underlie the problems of sexual and reproductive health in adolescents and young people.
\end{abstract}

Keywords: health's communication; sexual and reproductive health; state of the art; Mexico. 


\section{Introducción}

E I presente artículo tiene el objetivo de elaborar un estado del arte sobre la comunicación para la salud sexual y reproductiva (SSyR) de adolescentes y jóvenes en México. De ahí que tomemos en consideración la producción científica en torno a la comunicación sobre el embarazo adolescente, las Infecciones de Transmisión Sexual (ITS), el Virus de Inmunodeficiencia Humana y el Síndrome de Inmunodeficiencia Adquirida (V/H/Sida).

Los estados del arte son revisiones bibliográficas de distintas fuentes (investigaciones, reportes e informes de instituciones y organizaciones diversas, públicas 0 privadas, autónomas, artículos de revistas especializadas, libros de autor y compilaciones, tesis de pregrado y posgrado) que permiten encontrar antecedentes y condiciones académicas de un objeto de estudio para determinar su estado de conocimiento, así como las formas y estrategias reflexivas en las que se ha observado y analizado; ello implica hacer un balance de los principales avances y retrocesos de su estado de la cuestión, con base en los aportes, tendencias y regularidades halladas. A decir de Londoño, Maldonado \& Calderón (2014, p.6):

El estado del arte le sirve al investigador como referencia para asumir una postura crítica frente a lo que se ha hecho y lo que falta por hacer en torno a una temática o problemática concreta, para evitar duplicar esfuerzos o repetir lo que ya se ha dicho y, además, para localizar errores que ya fueron superados.

Como parte de la revisión bibliográfica, consultamos -en su mayoría-artículos cientificos en revistas indexadas, así como libros y tesis de pregrado y posgrado. Entre las revistas mexicanas consultadas se encuentran las siguientes: Revista de Estudios sociológicos, Revista de Salud Pública de México', Acta de investigación psicológica, Gaceta Médica de México, Estudios sobre las Culturas Contemporáneas, Papeles de Población, Comunicación y Sociedad, Revista de Salud Mental, Revista de Estudios Fronterizos, Revista de Estudios Sociales, Revista de Investigación en Tecnologías de la Información y Revista de Psicología y Salud.

También revisamos publicaciones regionales del continente americano, entre estas estuvieron la Interamerican Journal of Psychology, con sede en Florida, Estados Unidos; la Revista Diálogos de la Comunicación de la Federación Latinoamericana de Facultades de Comunicación Social (FELAFACS); la publicación oficial

\footnotetext{
1 Es la revista donde se obtuvieron más artículos, el $32 \%$ del total de las publicaciones revisadas.
}

de la Organización Panamericana de la Salud (OPS): la Revista Panamericana de Salud Pública, con sede en Washington D. C., Estados Unidos; y la Revista Latinoamericana de Ciencias Sociales, Niñez y Juventud, con sede en Manizales, Colombia; todas representativas de la región de América Latina. Asimismo, hallamos textos sobre la problemática en México, publicados en dos revistas brasileñas, la Revista de Saúde Pública, editada desde Sao Paulo y Cadernos de Saúde Pública, de Río de Janeiro; y en la Revista Española de Comunicación en Salud, de la Universidad Carlos III de Madrid.

Accedimos a la plataforma Academia.edu (internacional), el Repositorio Institucional (Rel) del Instituto Tecnológico de Occidente (ITESO) de México y la base de datos de la Guttmacher Institute de la Organización Mundial de la Salud (OMS), la Biblioteca Cientifica Electrónica en Línea (SCIELO, por sus siglas en inglés), La Red de Revistas Científicas de América Latina y el Caribe, España y Portugal (Redalyc) y la base de datos de revistas especializadas en biomédica, Mediagraphic.

El análisis de la información parte de los componentes de la efectividad de la comunicación, los paradigmas que se asumen, el lugar de la información en dichos procesos, el accionar de las instituciones como "emisores" en el proceso comunicativo, y desde el otro lado, los públicos a los que va dirigido: jóvenes, adolescentes, familiares, profesores, etc.

También hacemos alusióna los discursos de sexualidad y los enfoques de educación sexual que subyacen en los esfuerzos realizados, además de la influencia de las teorías del comportamiento en los programas que se llevan a cabo. Por otra parte, incluimos el conocimiento generado sobre los enfoques estructurales de vulnerabilidad dentro de estos programas, como elemento imprescindible para la efectividad de los mismos.

\section{Método}

Para el análisis de los textos realizamos un estudio sistemático de revisión de tipo narrativo, con el fin de evaluar, interpretar y sintetizar las características, resultados y hallazgos de dichas producciones académicas en el campo de nuestro interés. A decir de Rychetnik, Hawe, Waters, Barrat \& Frommer (2004, citado en Caballero, 2006, p.64), el estudio sistemático narrativo permite "generar una síntesis descriptiva e interpretativa de los hallazgos para obtener una visión panorámica de las evidencias". Esto nos permitió sistematizar los conocimientos que se han generado sobre la problemática en México en sentido general, pero 
haciendo énfasis en la manera en que se ha comprendido y tratado la temática, sus concepciones, modelos, discursos, enfoques, propuestas, aciertos y limitaciones

La revisión la focalizamos al espacio mexicano, con el fin de proponer una mirada particularizada del país objeto de estudio. El principal criterio de selección de los textos fue que incluyeran conocimientos acerca de los programas, planes y estrategias de comunicación, información y educación de la SSyR en México, sin importar si estaban publicados dentro o fuera del país.

Asimismo, la búsqueda tuvo un recorte temporal de 1994 a 2018. Ello estuvo sustentado en que en 1994 se realizó la Conferencia Internacional de Población y Desarrollo (CIPD), la cual motivó a los países a convertir la problemática en un asunto gubernamental, trayendo consigo una mayor proliferación de publicaciones sobre la temática; y hasta el 2018 (inclusive), serían los primeros 25 años después de la conferencia.

Las palabras clave utilizadas en la búsqueda fueron las siguientes: salud sexual y reproductiva, comunicación, educación, información y promoción de salud, riesgo sexual, conducta sexual de riesgo, comportamiento sexual, fuentes de información, programas de intervención, prevención, embarazo, ITS, VIH, Sida, métodos anticonceptivos, condón, adolescentes y jóvenes.

\section{Resultados}

De la búsqueda, resultaron 36 textos que cumplían los requisitos, de ellos: 2 (6\%) libros impresos, el resto provenía de material digital, $2(6 \%)$ tesis y $32(88 \%)$ artículos científicos publicados en revistas indexadas. Revisamos textos de 1994 a 2018, de los cuales, más del $45 \%$ han sido publicados en los últimos diez años (20092018), el $40 \%$ entre 2000 y 2008 , y el $14 \%$ en la década de 1990 (1994-1999).

Las investigaciones han estado más centradas -en este orden- en adolescentes y jóvenes (44\%), estudiantes $(27 \%)$, mujeres $(18 \%)$, intelectuales o dirigentes de instituciones $(12 \%)$, trabajadores de salud $(9 \%)$, padres y madres $(9 \%)$, e indígenas (3\%). Otras manejaron textos periodísticos o publicaciones oficiales (18\%) y en el caso de los ensayos teóricos, no se enfocaban en sujetos específicos, sino en las teorías en sí (27\%).

Los estudios declararon una metodología cualitativa (49\%), cuantitativa (40\%) o una perspectiva mixta (11\%) que mezclaba ambas. En cuanto al tipo de diseño experimental (11\%) o no experimental $(89 \%)$, es válido aclarar que en algunos casos tuvimos que deducirlo porque en la investigación no se declaraba. Esto guarda relación con resultados de otros estudios similares, en cuyos artículos analizados "no se especifica metodología alguna" (Terrón, et al., 2017, p.165).

Los tipos de muestreo fueron: no probabilísticos $(51 \%)$ y probabilísticos $(23 \%)$, en dependencia de las perspectivas metodológicas asumidas. Algunos contenían ensayos teóricos (26\%), y asumían un muestreo teórico o no expresaban ningún tipo de muestra. Por otra parte, los niveles de análisis fueron en su mayoría descriptivos $(75 \%)$, exploratorios (17\%) y correlacionales (9\%). Asimismo, sobresalieron los de tipo transversal (71\%), en oposición a los longitudinales (17\%).

En cuanto a los métodos y técnicas que aparecieron en la revisión bibliográfica, resultaron como más recurrentes: el análisis estadístico (37\%), seguido del análisis de contenido (17\%), los métodos teóricos Histórico-lógico e Inductivo-deductivo (11\%), la etnografía (11\%), la teoría fundamentada $(6 \%)$, el análisis del discurso (6\%), el interaccionismo simbólico (6\%), los estudios de caso $(3 \%)$ y el análisis de la dinámica contextual $(3 \%)$. Además, algunos se auxiliaron de programas de análisis cualitativos como el software NVivo 7 y Atlas.ti, mientras los cuantitativos utilizaron SPSS, AMOS o Excel.

El instrumento de registro privilegiado fue la entrevista $(29 \%)$, luego el cuestionario (26\%), el análisis de las encuestas nacionales $(24 \%)$, el análisis documental $(17 \%)$, los grupos focales $(9 \%)$ y las observaciones participantes y no participantes (9\%). En sentido general, utilizaban una variedad de métodos, técnicas e instrumentos, incluso algunos hacían combinaciones en dependencia de las metodologías que asumían, de acuerdo con los objetivos y resultados propuestos.

En los textos revisados pudimos encontrar distintos enfoques de las disciplinas de las Ciencias Sociales como la Psicología social, la Sociología, las Ciencias de la Educación y de la Comunicación, y los Estudios de Población, principalmente. Sin embargo, los enfoques teóricos más notables fueron los de educación sexual, las teorías del comportamiento, las teorías de la sexualidad, los estudios de género, la antropología cognitiva, las representaciones sociales, el estigma y la discriminación, así como la vulnerabilidad social y sexual.

\section{Discusión}

La discusión está organizada en seis apartados, cuyos límites hemos fijado en función de los grupos y tendencias investigativas encontradas. El primer apartado incluye la actuación de las instituciones, sus particularidades internas e influencias externas. El segundo presenta cómo se maneja la información, qué efectos tiene y 
qué relación guarda con los conocimientos, actitudes, creencias y expectativas de adolescentes y jóvenes. El tercer apartado trata sobre la pertinencia de inmiscuir a varios actores sociales y colocar a los sujetos como protagonistas del proceso comunicativo. El cuarto incluye los planteamientos que demuestran cómo la comunicación se hace eco de los discursos sociales conservadores sobre la sexualidad. El quinto sintetiza las ideas sobre la efectividad de la comunicación, haciendo especial énfasis en su evaluación. Por último, el sexto apartado trata la vulnerabilidad social y su relación con la atención a la SSyR.

\section{Las instituciones mexicanas: sus ataduras} internas y externas

La SSyR ha sido una problemática social de amplia cobertura institucional y gubernamental, con mayor interés internacional después de la CIPD de 1994. En este apartado presentamos una caracterización del accionar institucional mexicano que da cuenta de las características institucionales que lo condicionan en los ámbitos interno y externo.

Existen investigaciones que demuestran que la educación sexual ha recibido la oposición de actores conservadores como la Iglesia, cuyas "posiciones varían del rechazo al condicionamiento". Estos actores, "han formado redes que les confieren poder económico y político significativo", ante el cual, "el Estado ha cedido a algunas demandas" tanto de las instituciones como de sus opositores, como es el caso de la modificación de libros de textos gratuitos de la enseñanza de la sexualidad en las escuelas secundarias y de bachilleratos mexicanas (Granados, Nasaiya, \& Torres, 2007, p. 543).

Por otra parte, según Amuchástegui (2017, p. 343), "la respuesta a la epidemia del VIH en mujeres está sostenida por el paradigma de la vulnerabilidad que domina la gobernanza neoliberal". Por ello, "una organización gubernamental del sufrimiento distribuye recursos entre 'poblaciones clave'" y de esa manera, "orientada por la regulación moral de la sexualidad, funciona simultáneamente como razón de ser y camisa de fuerza de las organizaciones feministas y de mujeres que viven con $\mathrm{VIH}$, dificultando alianzas entre ellas". La autora añade que esto conlleva a que las mujeres adopten "múltiples formas de resistencia que sugieren la necesidad de cuestionar su representación como sujetos impotentes; y de problematizar, como tarea del feminismo, la dicotomía vulnerabilidad-resistencia" (Amuchástegui, 2017, p. 343).

En este sentido, encontramos que el colectivo de autores de Santos et al. (2003), habla de los retos que enfrentan las instituciones en México. Al respecto, apuntan que "las acciones en salud tienen que orientarse en diferentes esferas", y mencionan tres:

(...) la primera de ellas es vencer las causas prevenibles con el concierto social de la respuesta organizada2; la segunda, hacer atractiva la atención con un cambio de paradigma de servicios fijos a servicios que se ofrezcan en las escuelas y los centros de trabajo; el tercer nivel de respuesta es el cambio de enfoque, pasar de la simple atención a la salud a la atención enfocada no sólo a riesgo sino a resiliencia y desafío, fortalecer la economía y la producción, [para] romper los ciclos de repetición entre las generaciones de paternidad adolescente (2003, p.151).

Aunque algunos de estos elementos ya se encuentran obsoletos -porque han pasado casi quince años de la publicación del artículo-, otros continúan vigentes y reflejan cuánto se ha avanzado y cuánto no. Es notable que desde entonces, los autores vienen proponiendo la necesidad de un abordaje holístico de la sexualidad que "haga frente a los riesgos a los que se encuentran expuestos" los sujetos (Santos et al., 2003, p.151).

También otros estudios hablan de las barreras políticas de las instituciones. Juárez \& Gayet (2005, p.215) destacan que, entre estas, figuran "la falta de estabilidad de los programas, derivada de los cambios de funcionarios en las estructuras de mando y decisión". Dicha inestabilidad se debe a que las instituciones se organizan de manera centralizada y muchas veces el liderazgo se concentra en determinados sujetos, de cuya presencia/ausencia depende la continuidad de los proyectos.

En el tema puntual de la comunicación llevada a cabo por instituciones de salud, Gumucio (2010) destaca un conjunto de regularidades que caracterizan -y afectan negativamente- la actuación institucional. Sobre ello señala que:

Preocupa sobremanera la distancia entre el discurso institucional o gubernamental sobre el tema específico de la comunicación, así como las acciones que se llevan a cabo, que muestran no solamente una falta de comprensión sobre lo que es la comunicación, sino también la incapacidad de planificarla y de pensarla con una perspectiva estratégica (Gumucio, 2010, p.90).

Aparece la falta de planificación estratégica de las instituciones como un impedimento en su labor: "pocas organizaciones se han dotado de una política

2 Colaboración entre las instituciones que se relacionan con una determinada problemática social, para atenderla de manera conjunta. 
de comunicación". Eso lleva a la improvisación y al estancamiento en temáticas a veces trilladas, pero poco resueltas. "La carencia de una política, es decir, de una visión institucional clara y consensuada sobre la comunicación, les impide diseñar estrategias que apoyen de manera eficaz los programas de desarrollo (de salud y otros)" (Gumucio, 2010, p.91).

Aunque las instituciones reconocen la importancia de la comunicación y la participación, en la realidad, los programas y estrategias institucionales de salud se ven desprovistas de estas. Gumucio (2010, p.90), denuncia que la promoción institucional de la salud a veces se confunde con "la visibilidad institucional y en el mejor de los casos la diseminación masiva de información a través de mensajes". Esto hace que no se lleguen a concretar procesos de comunicación en salud donde la institución y los sujetos se involucren activamente.

Estos textos evidencian de las condiciones del accionar institucional en México, donde la moral conservadora de organizaciones sociales dominantes influye en el tratamiento de las problemáticas de SSyR. Además, prevalecen formas de organización y liderazgo que vuelve omnipotente a determinados sujetos, de cuyo poder depende lo que se hace; así como la falta de visión estratégica de la comunicación que impide un tratamiento eficaz de la problemática.

\section{Del silencio al estigma en la información institucional}

Un área de especial interés en la producción científica de la comunicación sobre SSyR es la generación y circulación de la información, cómo se maneja, qué efectos tiene y cómo se relaciona con los conocimientos, actitudes, creencias y expectativas de sus públicos adolescentes y jóvenes.

Hace más de veinte años, Rico, Bronfman, \& RioChiriboga (1995, p.652), sobre el VIH, apuntaban que "se sabe que la educación es un punto central en el combate a la epidemia, pero también que el conocimiento que se deriva de la pura información no basta para modificar comportamientos de riesgo". Posicionados en las teorías del comportamiento, los autores agregan que "es indispensable desarrollar intervenciones que toquen aspectos más profundos en los individuos a los que se quiere llegar". También reconocen que "las campañas masivas no cambian de manera importante los comportamientos, pero son útiles para mantener un estado de alerta y para crear el clima que haga aceptable otro tipo de intervenciones más puntuales y específicas". De esa manera, los autores han dejado en claro la pertinencia de segmentar los públicos y destinar esfuerzos de comunicación hacia grupos específicos y no hacia la población en general como se solía hacer durante esos años (Rico, Bronfman \& Rio-Chiriboga, 1995, p.652).

Caballero, Villaseñor \& Hidalgo (1997, p.351), indagando sobre las fuentes de información en relación con los estratos sociales de los usuarios y sus niveles de conocimiento, descubren que "hay desigualdades por estratos socio-económicos en la relación de las fuentes informativas y los niveles de conocimientos sobre VIH/Sida" que tienen los sujetos. De igual manera, "la información de periódicos y profesores tuvo relación directa con el nivel de conocimientos". Con base en ello, proponen "fortalecer y desarrollar acciones educativas por fuentes masivas e interpersonales, con énfasis en estratos bajo y marginados" (Caballero, Villaseñor, \& Hidalgo, 1997, p.351).

Otro estudio sobre las preferencias de los adolescentes mexicanos para hablar de Sida, demuestra que "el nivel social al que pertenecen (...), su condición de actividad sexual y laboral, y el sexo, parecen determinar la elección del interlocutor para hablar del Sida". Ello está sustentado en que "los hombres jóvenes hablan del Sida con su padre más que las mujeres, y las mujeres más con su madre". Del mismo modo, "los sexualmente activos hablan más con los amigos, y algo menos con los maestros, que los sexualmente inactivos" (Gayet et al., 2002, p.122).

En cuanto a los contenidos que circulan, Lozano, Torres, \& Aranda (2008) apuntan que "existe abundante información descriptiva en torno al padecimiento sobre la transmisión del VIH: es producida por un virus, es mortal y no tiene cura". La investigación sobre las concepciones culturales del VIH/Sida, arrojó que en el discurso de los jóvenes prevalecen "primero, elementos alusivos a un fatalismo ineludible y sentimientos que derivan del mismo; segundo, incluye conceptos biológicos; y tercero, considera cuestiones sexuales y señalamientos morales con respecto a las prácticas sexuales" (p.740). Estas concepciones demuestran la influencia en los jóvenes de un discurso biologicista que ha marcado la atención a la epidemia y que ha promovido estigmatización y rechazo hacia los sujetos afectados.

Varias investigaciones encontradas han tratado la temática de la información desde los proveedores o prestadores de servicios de salud. Un estudio reveló que, en los ambientes sanitarios existe discriminación hacia las personas seropositivas, a pesar de que se capacita sobre la epidemia. La investigación asienta que, "la percepción de que los hombres que tienen sexo 
con otros hombres y las personas que realizan trabajo sexual deciden sus prácticas sexuales, establece la división entre víctimas inocentes y culpables e influye en la estigmatización y discriminación en los servicios" (Infante et al., 2006, p.141).

El asunto es que la capacitación se realiza desde un enfoque de la sexualidad que contiene un discurso conservador, que clasifica a los sujetos por su comportamiento, en función de sus prácticas "irresponsables" y por lo tanto censuradas por la moral. En tal sentido, Infante et al. (2006, p.142) proponen que:

El diseño de medidas para disminuir el estigma y la discriminación relacionados con el VIH/Sida exige la inclusión del debate ético sobre los derechos humanos y un enfoque estructural de las condiciones sociales que rebase la noción de comportamientos de riesgo.

Castro \& Arellano (2010, p.211) señalan que "aunque es una disposición legal y ética, los prestadores de salud no ofrecen información, lo que genera incertidumbre ante procesos desconocidos y obstaculiza una adecuada toma de decisiones, que se complica con componentes emocionales y valorativos". Sin embargo, Campero et al. (2010, p.68) apuntan que:

Cuando las $\mathrm{PVVIH}^{3}$ logran recibir de sus compañeros de grupo y/o proveedores de salud información certera y clara, aumenta la seguridad en sus prácticas y es más factible para ellos adueñarse de sus derechos sexuales y reproductivos.

Campero et al. (2010) aseguran que una de las limitaciones de la prevención, por parte del personal de salud y de los grupos de apoyo a las personas seropositivas, es que enfatizan el uso del condón en las relaciones sexuales, para evitar la infección del otro o la reinfección de las personas seropositivas. "Este trabajo hace explícita, la necesidad no sólo de hablar del condón sino de trabajar con hombres y mujeres sobre estrategias de negociación del uso de métodos de protección en diferentes escenarios" (Campero et al., 2010, p.68).

También se ha hablado del lugar que ocupan determinados actores sociales en la generación y difusión de información. Romero (2012) tiene una investigación sobre las parteras como personajes clave en el empoderamiento de las mujeres en el medio rural. Dice el autor que, "son una autoridad moral de peso sufriente dentro de sus comunidades como para inducir cambios en patrones de conducta machista adversos en la difusión de la planificación reproductiva" (Romero, 2012, p.310). Esta constituye una de las posibles estrategias a seguir, para

$\overline{3 \text { Personas que viven }}$ con VIH. que las instituciones lleguen, de manera efectiva, a una población que no es fácil de acceder por otras vías (redes sociales, medios masivos de comunicación, etc.).

Otra recomendación es ajustar los mensajes a las competencias comunicativas de los sujetos. Caballero (2016), en un estudio con jóvenes indígenas jornaleros migrantes (JIJM), concluye que la efectividad de los mensajes dependerá de que se adapten "a la estructura homogénea del dominio cultural de los JIJM”. Además, se debe seguir una "estrategia por tipos de parejas sexuales (...) para prevenir el riesgo en relaciones basadas en parejas sexuales primarias, como otra para prevenir el riesgo en relaciones con parejas sexuales casuales. [Y] según la etnicidad de las parejas sexuales [indígenas o no]" (Caballero, 2016, p.208). Teniendo en cuenta que existen diferencias sustanciales cuando los JIJM tienen parejas casuales o primarias, y si estas son indígenas o no.

Los principales aportes de estos autores radican en sacar a la luz los contenidos biologicistas y moralistas presentes en la información, en especial sobre el $\mathrm{VIH} /$ Sida, los cuales dan al traste con un sentido fatalista que en nada ayuda a la atención de la epidemia, porque generan estigmatización y discriminación hacia las personas seropositivas. También se subraya la importancia de recurrir a las parteras, como vía idónea para la promoción de salud, así como crear mensajes para grupos específicos de sujetos, basados en sus características sociodemográficas, sus preferencias de interlocutores y de medios de comunicación: tradicionales y alternativos, masivos e interpersonales.

\section{Escuela y familia: la interconexión entre las instituciones y sus públicos}

El cambio de paradigma de comunicación de transmitir a compartir, "poner en común", ha demostrado la urgencia de operar cambios en la manera en que tradicionalmente se han desarrollado los procesos de comunicación, donde los sujetos están ubicados en dos polos: emisores 0 receptores. A continuación, relatamos cómo la promoción de la salud demanda inmiscuir a varios actores sociales, distribuir simétricamente los roles para colocar a los sujetos como protagonistas del proceso.

Stern (2004), en correspondencia con otros autores (Mensch, Bruce, \& Greene, 1998; Kirby, 2001), recomienda "estimular y apoyar la organización de actividades colectivas para mujeres jóvenes (...); además de ofrecer a las jóvenes un espacio para reunirse e intercambiar ideas, preocupaciones etc." (p. 154).

Por su parte, Campero et al. (2013, p.149) explican la conveniencia de "favorecer el vínculo de la comunidad con las escuelas, los centros de salud y otras instancias" 
al tiempo de "reforzar la colaboración de distintas dependencias gubernamentales, organizaciones de la sociedad civil y agencias internacionales" como elementos significativos para la efectividad de su labor.

Varios estudios (Gayet et al., 2002; Atienzo, Campero, Estrada, Rouse, \& Walker, 2011; Bárcena, Robles, \& Díaz-Loving, 2013) coinciden en la impostergable incorporación de los miembros de la familia como sujetos activos de la comunicación.

Gayet et al. (2002, p.122) se percataron "que a mayor escolaridad del padre [o madre], los jóvenes tienen más interlocutores y hablan más sobre el Sida en el ámbito familiar, comparados con los que tienen padre [o madre] sin escolaridad". Esto corrobora la importancia de conocer el medio familiar en que viven los sujetos a quienes van dirigidas las estrategias, así como ajustarla en función de sus condiciones.

La investigación de Atienzo et al. (2011, p. 160) arrojó que "las intervenciones educativas que incluyen a padres y madres de familia favorecen comportamientos sexuales saludables en los adolescentes". De ahí la pertinencia de colocarlos como un público especial dentro del proceso comunicativo, con el fin de promover el diálogo con sus hijos sobre la sexualidad y su cuidado, a tiempo y oportunamente.

En correspondencia, Campero et al. (2013, p. 304) proponen "involucrar a los padres [y madres] de familia en la educación sexual de sus hijos, invertir en el entrenamiento y sensibilización de docentes y proveedores de servicios de salud".

Martell et al. (2018, p.15), en una investigación sobre representaciones sociales, hallaron que "la figura materna resultó tener una función predominante en la comunicación incluso en los hombres, además de que transmite valores e impone límites".

Bárcena, Robles, \& Díaz-Loving (2013, p.965) encontraron que, "los hijos de los padres [y madres] que tuvieron conocimientos más adecuados sobre cómo se usa un condón, fueron los que reportaron comunicarse más con sus padres [y madres] sobre cómo protegerse", lo cual demuestra la necesidad de que la familia esté preparada, y la efectividad del intercambio de información de jóvenes con éstos. También señalan que "las expectativas positivas de los padres [y madres] hacia la comunicación con sus hijos sobre temas sexuales, favorecieron que sus hijos tuvieran menos creencias negativas hacia el uso del condón". Sin embargo, "dichas creencias se incrementaron cuando los padres [y madres] tuvieron mayor nivel de estereotipos hacia el cuidado de la salud sexual de sus hijos". Es decir, el estudio encontró una influencia directa entre las creencias de jóvenes y sus familias, demostrando así que la familia tiene un papel preponderante en la transmisión de conocimiento a los hijos, el cual es necesario aprovechar para, a través de unos, incidir en los hábitos de autocuidado de los otros (Bárcena, Robles, \& Díaz-Loving, 2013, p.965).

Lo mismo sucede con el uso del condón en la primera relación sexual, la familia es capaz de incidir en esto, sin embargo, en ocasiones carecen de herramientas para hacerlo. Sustentados en sus hallazgos, los autores recomiendan que "los programas de intervención orientados a la prevención de problemas de salud sexual deben dirigirse [también] a jóvenes sin experiencia sexual y contemplar la participación y capacitación de los padres [y madres] en la promoción de conductas preventivas de sus hijos" (Bárcena, Robles, \& Díaz-Loving, 2013, p.965).

Además de la familia y la escuela como principales influyentes en la toma de decisiones respecto a asuntos de sexualidad, el estudio de Peña et al. (2018, p.170) arrojó que, los adolescentes encuestados se informan cada vez más por el ciberespacio.

Las redes sociales fueron las más demandadas por los adolescentes en un $71.6 \%$ y el facebook ocupó el $77.3 \%$, seguido de Instagram (17\%). El medio de comunicación ajeno a la Internet más utilizado para informarse sobre los métodos anticonceptivos fueron las revistas o folletos en un $57.1 \%$.

Este resultado contrasta con el de otros estudios con jóvenes en México, donde sus autores encontraron que "el recurso de utilización de Facebook para capacitarse en salud resulta poco recurrente en este grupo social" (Santamaría, Catalán \& Merced, 2016, p.15).

Sin embargo, las investigaciones revisadas aquí, demuestran que la promoción de la salud no puede ser asunto únicamente de las instituciones con este encargo social. Es preciso promover la comunicación asertiva en adolescente y jóvenes, e involucrar a aquellos sujetos que tienen indiscutiblemente una influencia sobre éstos, como es el caso de la familia y la escuela. De ahí la importancia de incluirlos en el proceso de comunicación como conectores entre los programas de intervención y sus públicos.

\section{El discurso de la sexualidad o la reproducción de la censura}

Hay varios elementos que atentan contra la atención a la SSyR de adolescentes y jóvenes, entre ellos están los discursos sociales sobre la problemática. En este apartado concentramos los planteamientos que demuestran cómo 
la comunicación hace eco de los discursos sociales conservadores sobre la sexualidad y su salud.

Según Juárez y Gayet (2005, p.216), en México, "los mayores constreñimientos parecen residir en la moral conservadora de la población, donde se mezclan preceptos católicos e indígenas". Esta moral termina siendo perpetrada, socializada y reproducida en espacios familiares, escolares y mediáticos. De esta manera, "la censura social sobre la sexualidad funciona como una barrera para la transmisión abierta de información al respecto" (Juárez \& Gayet, 2005, p. 216).

Un aporte significativo del discurso social sobre el VIH en jóvenes de México, lo realiza Collignon (2009). La autora apunta que:

El discurso social sobre el VIH ha remarcado el valor negativo de prácticas sexuales que se encuentran en los límites del modelo de sexualidad imperante y legitimado en México, prácticas que hablan de una sexualidad no exclusivamente heterosexual, monogámica, matrimonial ni reproductiva (Collingon, 2009, p.12).

Esto hace que la percepción del riesgo se distorsione con base en los significados de la sexualidad socialmente permitida o prohibida, porque establece una relación directa entre las posibilidades de infección con ciertas prácticas (frecuentes, promiscuas, peligrosas, riesgosas, inmorales, excesivas, etc.) y ciertas personas (prostitutas, promiscuas, infieles, inmorales, homosexuales, bígamos, liberales, etc.) (Collignon, 2009). Según la autora:

Esto imposibilita que los sujetos sociales identifiquen las condiciones objetivas de riesgo de infección por $\mathrm{VIH}$ (desprotección de las prácticas sexuales), y den mayor peso a la naturaleza de las personas, la frecuencia de las relaciones sexuales y el tipo de compañía sexual con quien se establece la relación (Collingon, 2009, p.12).

De esa manera, se culpabiliza la sexualidad, se estigmatiza el sexo, y el riesgo deja de ser lo objetivo, porque el discurso se concentra en la práctica y la persona censuradas. Es válido recordar que la sexualidad ha funcionado históricamente como dispositivo de poder para introducir el miedo en las relaciones socioafectivas y eróticas. A lo que la autora añade que, "la modernidad ha querido convertir al Sida en un dispositivo de control de la sexualidad" (Collington, 2009, p.12). Además, con una marcada influencia foucaultiana, la autora asevera que:

El dominio de y sobre el cuerpo ya no parece imponerse desde fuera; los mecanismos de socialización y la institucionalización del poder se han combinado para que el dominio sobre el propio cuerpo se ejerza por propia voluntad y por propia mano. El control invade, el control permea a los sujetos sociales y logra instalarse con relativa fuerza en los entresijos de la vida cotidiana (Collington, 2009, p.13).

Otro estudio interesante lo realizan Sánchez, Terrón, \& Lozano (2016), sobre la estigmatización del VIH en el discurso periodístico. Ahí encuentran dos tipos de estigmatización: explícita e implícita. Apuntan que la primera ha disminuido considerablemente en los últimos años, aseguran que los diarios utilizan un lenguaje neutro y no relacionan la enfermedad con la muerte, ni utiliza datos alarmistas: "la información que los medios de comunicación ofrecen (...) han permitido eliminar algunos mitos relacionados con el VIH y desvincular, en cierta medida, al Sida de la muerte" (p.94).

Por su parte, "la estigmatización intrínseca queda manifiesta en la escasa participación que tienen las personas afectadas en la producción de noticias, por temor a ser identificados con repercusiones negativas en su vida laboral y social" (Sánchez, Terrón, \& Lozano, 2016, p.94). El mismo estudio marca pautas de acción para mejorar el tratamiento periodístico del VIH/Sida en los diarios mexicanos. Propone hacer hincapié en:

a) la prevención y la educación sexual utilizando un lenguaje claro y preciso sin tendencias moralistas o de normalización, esto con el fin de erradicar los estigmas asociados a la diversidad sexual; b) las opciones de tratamiento que tienen las $\mathrm{PVVIH}$; c) las formas que tienen para defender sus derechos las PVVIH o sus allegados cuando son víctimas de discriminación, así como las instituciones y organismos en las que pueden encontrar diversos apoyos para mantener su tratamiento y mejorar su calidad de vida (Sánchez, Terrón, \& Lozano, 2016, p.95).

Otros de los elementos que están incidiendo en la efectividad de la comunicación son precisamente los enfoques de la sexualidad, desde los cuales se atiende la SSyR de adolescentes y jóvenes. Una práctica altamente difundida ha sido silenciar la sexualidad humana, sustentado en la creencia de que el tratamiento de estas temáticas incita a los individuos a tener prácticas sexuales. Ante este enfoque, Pick de Weiss et al. (1994, p.30), señalan que "no solo es importante ofrecer el programa, sino hacerlo antes de que los jóvenes empiecen a tener relaciones sexuales, (...) en edades tempranas".

Stern (1997), sobre los enfoques imperantes de la sexualidad en México, se cuestiona: "¿no será que muchos de los problemas que llevan al embarazo adolescente y a los que éste conduce se derivan de la 
manera como hemos valorado la sexualidad premarital y, particularmente, sancionado la sexualidad adolescente?". Su investigación le permitió reconocer que:

La sanción negativa obstaculiza el acceso a la información, la educación y la preparación para ejercer la sexualidad de una manera placentera y responsable, de modo que buena parte del problema estriba en la manera como los adultos calificamos el fenómeno; en la forma como las instituciones sociales -la familia, la escuela, las instituciones religiosas, el Sector Salud, etcétera- lo interpretan y lo manejan (Stern, 1997, p.142).

De esta manera, este grupo de autores deja constancia de cómo el riesgo sexual está siendo consecuencia de discursos y enfoques de la sexualidad moralistas que encuentran en las instituciones sociales un mecanismo para sancionar la sexualidad y así obstaculizar su ejercicio pleno y responsable. Ello lo demuestra el hecho de que la sexualidad ha funcionado históricamente como un dispositivo de poder, de control y de generación de miedos que distorsiona la percepción del riesgo. Se hace imprescindible entonces, repensar las maneras en que se ha entendido la problemática y cómo se reproducen la estigmatización, la censura e incluso la invisibilización de la sexualidad.

\section{La evaluación y la (in)efectividad de la comunicación}

La efectividad del tratamiento de la SSyR ha sido un foco delirante en la reflexión teórica en esta área, debido a que se llevan a cabo acciones para enfrentarla, y no obstante, la situación desfavorable persiste e incluso aumenta, lo cual deja en evidencia la efectividad de lo que se hace. Aquí traemos a colación las ideas encontradas sobre esta cuestión, haciendo especial énfasis en la relación entre evaluación y efectividad de la comunicación.

Stern (2004) habla de los esfuerzos que se hacen en México para brindar información a adolescentes y jóvenes. Sin embargo, muchos de los programas no pasan de la mera cuestión informacional, incluso, ni siquiera en ese sentido son suficientes, pues señala que "se quedan muy cortos en términos de las necesidades de los distintos sectores de la población para posponer la maternidad o prevenir embarazos no deseados" (Stern, 2004, p.155).

En cuanto a la incorporación de temáticas de sexualidad en los currículos escolares, Gallegos et al. (2008, p.59), sostienen que "la intervención conductual representa un importante esfuerzo en la promoción de conductas sexuales seguras en adolescentes mexicanos". Este estudio experimental arrojó que fue efectiva la intervención en las intenciones de los adolescentes de usar condón y anticonceptivos a través del currículo escolar. Aunque aclaran que los "hallazgos son promisorios debido a la escasez de currículos basados en evidencia científica que hayan probado ser eficaces en la modificación de conductas sexuales de riesgo para VIH/Sida con adolescentes".

También se ha insistido en la urgencia de enfocar los esfuerzos hacia áreas y sujetos específicos. Lozano, Torres \& Aranda (2008, p.740) proponen que las "campañas deberían hacer énfasis en actividades de prevención específica como es el uso de preservativos. Y el contenido debería estar libre de elementos moralistas". Asimismo, Uribe et al. (2012) proponen tenerse en cuenta las diferencias de género:

Es necesario que se considere que las mujeres y los hombres tienen roles social y culturalmente construidos, los cuales influyen en su comportamiento sexual, por lo que se debe promocionar el uso correcto de condón con estrategias diferentes para hombres y para mujeres (Uribe et al., 2012, p.492).

Igualmente, Campero et al. (2013, p.304) señalan la importancia de tener en cuenta la multidimensionalidad de la sexualidad: "los programas de educación sexual para adolescentes deben partir de un enfoque incluyente, considerando aspectos biológicos, éticos, afectivos, sociales, culturales y de género".

Varios estudios concuerdan en que la efectividad está determinada por la no evaluación de los procesos, lo cual conduce a que no se tenga certeza del origen de los efectos obtenidos, y como consecuencia se repitan los mismos errores (Torres et al., 2006; DeMaría et al., 2009; Juárez et al., 2010; Campero et al., 2013).

Juárez y Gayet (2005, p.214) afirman que "existen numerosos organismos con programas dedicados a los jóvenes, pero sin coordinación operativa entre ellos". Esto hace que no exista un discurso coherente entre las diferentes instancias y que, en consecuencia, tributen hacia el mismo objetivo de manera aislada, inconexa, y por lo tanto, menos abarcadora y funcional.

Una investigación sobre el conocimiento, las actitudes y el uso de la Anticoncepción de Emergencia $(A E)$, describe "la necesidad de evaluar estrategias de prevención de VIH/Sida/ITS y embarazos no planeados que incluyan $A E$, de manera que generen evidencia sobre su efectividad en la prevención del embarazo adolescente y su posible incidencia en el riesgo ante las ITS" (Torres et al., 2006, p.214). Esto sucede porque no se llega a conocer si realmente los programas y estrategias consiguieron sus objetivos, si incidieron sobre 
la problemática o generaron algún cambio.

También, es importante observar el aumento o disminución en las estadísticas oficiales. Sobre ello, DeMaría et al. (2009, p.485) señalan que "los esfuerzos de evaluación de la efectividad de los programas deben contemplar desenlaces tales como marcadores biológicos (incidencia y prevalencia de ITS y embarazo) y no únicamente indicadores de conocimiento y comportamiento sexual auto-reportado".

Por otra parte, se ha hablado de la pertinencia de una adecuada coordinación y monitoreo de los programas en las escuelas, lo cual implica la capacitación de los maestros para hacer ese tipo de trabajo. A decir de Juárez et al. (2010), estos figuran como elementos esenciales para conseguir la eficacia de los programas y así refinarlos en adelante. Los autores proponen "implementar (...) un mecanismo formal y permanente de monitoreo y evaluación, para identificar aquellos programas que son exitosos y aquellos que tienen debilidades perennes" (Juárez et al., 2010, p.25).

También en el ámbito escolar, la investigación de Campero et al. (2013) concluyó recientemente que en México:

No existen evaluaciones formales, a nivel institucional, sobre los programas de educación sexual ni sobre la calidad de impartición de sus contenidos. Su evaluación aportará elementos sustantivos para la modificación del currículo de acuerdo con necesidades pertinentes y actuales para los adolescentes (Campero et al., 2013, p.304).

Estas publicaciones revisadas han revelado, desde diferentes posturas, los elementos que han estado incidiendo en la efectividad de la comunicación en la SSyR en México en los últimos años. Algunos de ellos ya resultan recurrentes en las investigaciones revisadas, como por ejemplo, la falta de planificación estratégica y de coordinación operativa de las instituciones, el direccionamiento hacia públicos generales y no específicos, la influencia de discursos sociales moralistas y la no incorporación de la escuela y la familia como sujetos relevantes de esta labor.

También, sobresale la importancia de la evaluación de los programas y estrategias de prevención, para conocer su efectividad, como mecanismo para rectificar, modificar y reajustar los planes, programas, proyectos y currículos escolares. Las investigaciones apuntan que la efectividad debe medirse no solo por el aumento de conocimiento que se logre en los sujetos, porque ello no es garantía de tener prácticas sexuales seguras; la evaluación de la efectividad debe contrastarse con las tendencias estadísticas de la población meta.

\section{Atender la vulnerabilidad: la deuda gubernamental}

Desde una perspectiva estructural de entender y atender las problemáticas de SSyR, varios autores han descrito el riesgo sexual como vulnerabilidad social, enfoque distinto a las conservadoras teorías del comportamiento. Desde esta postura, se entiende que el riesgo sexual está condicionado por procesos de segregación y marginación, que ubican a los sujetos en posiciones desventajosas dentro de la estructura social. En este último apartado concentramos las propuestas de la promoción en salud, desde la vulnerabilidad social.

Stern (2004, p.153) plantea que "el problema del embarazo adolescente necesita ser analizado dentro de los parámetros que lo rodean, tales como la transición demográfica, la desigualdad social y la pobreza, el ambiente político y los procesos vigentes de cambio social y cultural". Entender la problemática desde esta perspectiva, implica situarla no como meros acontecimientos aislados de la realidad, sino como un subproducto de procesos demográficos, económicos, políticos, sociales y culturales.

Un elemento estructural que condiciona el riesgo sexual es el nivel de escolaridad. Menkes \& Suárez (2003, p.1) logran demostrar que, "un nivel bajo de escolaridad femenino se asocia con un menor conocimiento y uso de métodos anticonceptivos, una menor planeación de la primera relación sexual y una edad más temprana en la iniciación sexual". En su conjunto, estas condicionantes se transforman en vulnerabilidad ante el embarazo y las ITS. De ahí la pertinencia de combinar las labores de información, educación y comunicación con otras de carácter económico, político y social que muevan a los sujetos de su condición de vulnerabilidad. A tono con ello, Villalobos et al. (2015) agregan en su estudio que:

En estos contextos es de suma importancia propiciar que la escuela sea generadora de cohesión social, con planes curriculares atractivos y adecuados a las necesidades especíicas de cada grupo social, así como favorecer incentivos por los que valga la pena continuar estudiando antes que realizar otra actividad (Villalobos et al., 2015, p. 142).

Ello es posible a través de políticas intersectoriales e interinstitucionales que incluyan la escuela, la comunidad, las familias, las instituciones de gobierno en las áreas de salud, educación, economía, los institutos de juventud y de la mujer, entre otras, como principales actores sociales que tienen como objetivo promover el ejercicio saludable de la sexualidad. Los autores también hablan de que las 
estrategias "sean sensibles y acordes a las necesidades locales y con pertinencia cultural y lingüística" (Villalobos et al., 2015, p.142).

Igualmente, aparece la violencia en las relaciones de noviazgo como una de las variables concomitantes del riesgo sexual. Estudios demuestran que las personas que son violentadas al mismo tiempo son vulnerables de contraer una ITS o embarazo no deseado. Fernández de Juan (2014, p.73) enfatiza que ambas conductas de riesgo deben ser tratadas en conjunto, lo cual demanda "la implementación de una eficiente, desprejuiciada y oportuna educación sexual, sobre todo en el ámbito escolar y con una perspectiva de género, de la que se carece en sentido general, en el país".

Estas investigaciones enriquecen la comprensión de la problemática y exponen la necesidad de tener en cuenta las múltiples dimensiones de la SSyR en los planes, programas y estrategias que se realizan en torno a ella. A decir de Caballero (2016, p.207), "la principal y más eficiente acción sería la modificación de los factores estructurales que condicionan la situación de la pobreza extrema, marginación, explotación y exclusión social". Es decir, no solo depende del esfuerzo humano y económico que se invierta en dichos planes, sino de promover simultáneamente, una transformación estructural.

\section{Conclusiones}

La revisión realizada nos permite llegar a la conclusión de que la labor de las instituciones que atienden la problemática en cuestión, está influenciada por condiciones externas como la imposición ideológica de la moral conservadora de los grupos de poder; y marcada por escenarios internos donde destacan las formas organizativas jerarquizadas, la falta de visión y planeación estratégica de la comunicación y la improvisación que ello trae consigo.

Los estudios revisados dejan clara la necesidad de abogar por la segmentación de los públicos, la producción de mensajes para grupos específicos y el auxilio de actores relevantes, como los prestadores de servicios de salud, familiares y docentes. Además, defienden el uso específico y combinado de medios de comunicación: tradicionales y alternativos, masivos e interpersonales, que promuevan una comunicación asertiva en los adolescentes y jóvenes, así como habilidades para negociar el ejercicio de una sexualidad responsable.

La información que circula es contenedora de aspectos biologicistas y moralistas que acentúan el sentido de victimización, culpabilidad, estigmatización y discriminación hacia los sujetos en riesgo, lo cual limita la atención efectiva a la SSyR. Esto proviene de un discurso social conservador que encuentra en la comunicación un espacio para ser institucionalizado, reproducido y legitimado.

También es posible encontrar en los textos revisados que la inefectividad de los procesos ha sido consecuencia de concepciones de comunicación y sexualidad sesgadas en la acción institucional. Además de estar determinada por la falta de evaluación de los planes, programas y proyectos con este fin. La evaluación constituye el recurso imprescindible para medir los logros, rectificar lo incorrecto y perfeccionarlo.

Los trabajos revisados asientan que asumir un enfoque de la vulnerabilidad social se convierte en una tarea impostergable para entender y atender eficientemente la SSyR de adolescentes y jóvenes en México. Es preciso tener en cuenta los procesos demográficos, económicos, políticos, sociales y culturales que atraviesan y condicionan que determinados sujetos se vuelvan más vulnerables ante el riesgo sexual. Ello demanda enfocar la mirada en otras variables concomitantes como la deserción escolar y la violencia en la pareja, de género e intrafamiliar. De esta manera, la inclusión de un enfoque de vulnerabilidad que promueva transformación estructural, debe ser la meta de cualquier institución con este encargo social.

Los hallazgos demuestran que es importante prestar atención a la relación comunicación-institución que aparece como uno de los principales ejes mediadores del tratamiento a la SSyR. Sería oportuno investigar a profundidad los discursos y enfoques que asumen sobre la sexualidad y la comunicación, además de la manera en que se organizan y llevan a cabo sus acciones. Asimismo, aparece el estudio de la efectividad de la comunicación como un área de oportunidad investigativa, debido a que las investigaciones revisadas dan cuenta recurrentemente de la necesidad de promover procesos de comunicación efectivos. Sin embargo, queda solo a nivel propositivo o enunciativo pues no abundan las investigaciones que asuman la efectividad de la comunicación como objeto de estudio propiamente.

De igual modo, resalta la pertinencia de focalizar la evaluación dentro de los procesos comunicativos, como elemento de vital importancia para conocer la efectividad social y cultural de la comunicación, cómo se concibe, qué mide, en qué momento, etc. También aprehender la manera en que se relacionan los distintos componentes del proceso comunicativo: la información que genera, cómo circula, qué contenidos privilegia, qué mensajes produce, qué efectos tiene; el lugar de los públicos en dicho proceso, cómo son concebidos, cómo 
se segmentan, qué protagonismo merecen, quiénes intervienen, cómo se convocan; también los medios de comunicación que se emplean, cuáles se privilegian, cómo se utilizan y combinan, etc.

Por último, los resultados del estado del arte justifican la importancia de desarrollar investigaciones que superen las teorías del comportamiento y se enfrasquen en la tarea de desentrañen los condicionamientos estructurales, que recrudecen las problemáticas de SSyR en adolescentes y jóvenes.

\section{Financiación}

El primer autor es becario de doctorado del Consejo Nacional de Ciencia y Tecnología de México y del Consejo Latinoamericano de Ciencias Sociales (2016-2020).

\section{Referencias Bibliográficas}

Amuchástegui, A. (2017). Gobernanza neoliberal en la epidemia del VIH/SIDA en mujeres en México: los efectos del paradigma de la vulnerabilidad. Estudios sociológicos, 35(104), 343-371. https://dx.doi. org/10.24201/es.2017v35n104.1511

Atienzo, E, Campero, L., Estrada, F., Rouse, C., \& Walker, D. (2011). Intervenciones con padres de familia para modificar el comportamiento sexual en adolescentes. Salud Pública de México, 53(2), 160-171. Recuperado de http://www.scielo. org.mx/scielo.php?script=sci_arttext\&pid=S0036$36342011000200009 \&$ Ing=es\&tlng=es

Bárcena, S., Robles, S., \& Díaz-Loving, R. (2013). El papel de los padres en la salud sexual de sus hijos. Acta de Investigación Psicológica, 3(1), 956-968. Recuperado de http://www.scielo.org. $\mathrm{mx} /$ scielo.php?script=sci_arttext\&pid=S200748322013000100005\&lng=es\&tlng=es.

Caballero, J. R. (2006). Factores de comportamiento asociados al riesgo de ITS y VIH en adolescentes y jóvenes mexicanos. revisión de estudios publicados entre 1986 y 2004. En J. R. Caballero, C. J. Conde, \& A. Villaseñor(Editores). ITS, VIH/SIDA en adolescentes $y$ adultos jóvenes. Guadalajara, México: Pandora.

Caballero, J. R. (2016). Sexualidad y riesgo de ITS y VIH/ SIDA en jóvenes indígenas jornaleros migrantes: hallazgos de un estudio mixto. En A. Cruz-Manjarrez, M. A. Chávez, \& A. I. Zermeño (comp.) Los jóvenes en el mundo actual. Deconstrucciones de las nuevas realidades. Colima, México: Enfoque Académico.

Caballero, J. R., Villaseñor, A., \& Hidalgo, A. (1997).
Fuentes de información y su relación con el grado de conocimientos sobre el SIDA en adolescentes de México. Revista de Saúde Pública, 31(4), 351-359. https://dx.doi.org/10.1590/S0034-89101997000400004.

Campero, C. L., Atienzo, E. E., Suárez, L. L., Hernández, P. B., \& Villalobos, H. A. (2013). Salud sexual y reproductiva de los adolescentes en México: evidencias y propuestas. Gaceta Médica de México, 149(3), 299-307. Recuperado de http://new.medigraphic.com/ cgi-bin/resumen.cgi?IDARTICULO=42887.

Campero, L., Kendall, T., Caballero, M., Mena A. L., \& Herrera, C. (2010). El ejercicio de los derechos sexuales y reproductivos: un estudio cualitativo de personas heterosexuales con VIH en México. Salud Pública de México, 52(1), 61-69. Recuperado de http://www. scielo.org.mx/scielo.php?script=sci_arttext\&pid=S003636342010000100010\&lng=es\&tlng=es.

Castro, M. C., \& Arellano, M. C. (2010). Acceso a la información de mujeres con VPH, displasia y cáncer cervical in situ. Salud Pública de México, 52(3), 207-212. Recuperado de http://www.scielo. org. $\mathrm{mx} /$ scielo.php?script=sci_arttext\&pid=S0036$36342010000300004 \& \operatorname{lng}=e s \&$ tIng=es.

Collignon, M. (2009). Construcción social de la sexualidad y el SIDA en el México contemporáneo. Renglones, 60, 1-15. Repositorio Institucional del ITESO. Recuperado de http://hdl.handle.net/11117/248

DeMaria, L. M., Galárraga, O., Campero, L., \& Walker, D. M. (2009). Educación sobre sexualidad y prevención del VIH: un diagnóstico para América Latina y el Caribe. Revista Panamericana de Salud Publica, 26(6), 485-493. Recuperado de https://www.ncbi. nlm.nih.gov/pmc/articles/PMC4720267/

Fernández de Juan, T. (2014). La educación sexual y de género vs el maltrato en la pareja. Escenario sobre la violencia en jóvenes de Baja California. Estudios Fronterizos, 15(1) 73-96. Recuperado de http:// www.redalyc. org/articulo.oa?id=53033724003.

Gallegos, E., Villarruel, A., Loveland-Cherry, C., Ronis, D., \& Zhou, Y. (2008). Intervención para reducir riesgo en conductas sexuales de adolescentes: un ensayo aleatorizado y controlado. Salud Pública de México, 50(1) 59-66. Recuperado de http:// www.redalyc. org/articulo.oa?id=10650109.

Gayet, C., Rosas, C. A., Magis, C., \& Uribe, P. (2002). Con quién hablan los adolescentes mexicanos sobre el SIDA. Salud Pública de México, 44(1), 122-128. Recuperado de http://www.scielo.org. 
$\mathrm{mx} /$ scielo.php?script $=$ sci_arttext $\&$ pid $=\$ 0036$ $36342002000200006 \&$ lng=es\&tlng=es

Granados, J. A., Nasaiya, K., \& Torres, A. (2007). Actores sociales en la prevención del VIH/SIDA: oposiciones e intereses en la política educativa en México, 1994-2000. Cadernos de Saúde Pública, 23(3), 535-544. https://dx.doi.org/10.1590/S0102$311 \times 2007000300012$.

Gumucio, A. (2010). Cuando el doctor no sabe. Comentarios críticos sobre promoción de la salud, comunicación y participación. Estudios sobre las Culturas Contemporáneas, 16(1), 67-93.

Infante, C., Zarco, A., Cuadra, S M., Morrison, K., Caballero, M., Bronfman, M., \& Magis, C. (2006). El estigma asociado al VIH/SIDA: el caso de los prestadores de servicios de salud en México. Salud Pública de México, 48(1), 141-150.

Juárez, F., \& Gayet, C. (2005). Salud sexual y reproductiva de los adolescentes en México: un nuevo marco de análisis para la evaluación y diseño de políticas. Papeles de Población, 11(1), 177-219.

Juárez, F., Palma, J. L., Singh, S., \& Bankole, A. (2010). Las Necesidades de Salud Sexual y Reproductiva de las Adolescentes en México: Retos y Oportunidades. Nueva York Guttmacher Institute. Recuperado de http://repositorio.gire.org. mx/bitstream/123456789/2206/1/NecesidadesSalud AdolescentesMexico_Guttmacher_ene2010.pdf.

Kirby, D. (2001). Emerging answers. Research findings on programs to reduce teen pregnancy. The National Campaign to Prevent Teen Pregnancy. Washington. Recuperado de https://files.eric.ed.gov/fulltext/ED456171.pdf.

Londoño, O. L., Maldonado L. F. \& Calderón, L. C. (2014) Guía para construir estados del arte. International Corporation of Network of Knowledge. Bogotá. Recuperado de http://www.colombiaaprende.edu. co/html/investigadores/1609/articles-322806_ recurso_1.pdf

Lozano, A F., Torres, T M., \& Aranda, C. (2008). Concepciones culturales del VIH/Sida de estudiantes adolescentes de la Universidad de Guadalajara, México. Revista Latinoamericana de Ciencias Sociales, Niñez y Juventud, 6(1), 739-768.

Martell, N. G., Ibarra, M. L., Contreras, G., \& Camacho, E. J. (2018). La sexualidad en adolescentes desde la teoría de las representaciones sociales. Psicología y Salud, 28(1), 15-24.

Menkes Bancet, C., \& Suárez López, L. (2003). Sexualidad y embarazo adolescente en México. Papeles de Población, 9(1), 1-31.

Mensch, B., Bruce, J., \& Greene, M. (1998). The uncharted passage. Girls' adolescence in the developing world. Population Council. New York. Recuperado de https://www.popcouncil.org/uploads/ pdfs/passage/passage.pdf.

Peña, G. M., Ruiz, A. L., Ley, S. V., Castro, J. J., Madrid, P. P., \& Apodaca, F. J. (2018). Patrones de comunicación dominantes en el conocimiento y empleo de los métodos anticonceptivos en adolescentes. Revista de Investigación en Tecnologías de la Información, 6(12), 170-180.

Pick de Weiss, S., Andrade-Palos, P., Townsend, J., \& Givaudan, M. (1994). Evaluación de un programa de educación sexual sobre conocimientos, conducta sexual y anticoncepción en adolescentes. Salud Mental, 17(1), 25-31.

Rico, B., Bronfman, M, \& Río, C. (1995). Las campañas contra el SIDA en México: ¿Los sonidos del silencio o puente sobre aguas turbulentas? Salud Pública de México, 37(1), 643-653.

Santamaría, C. D., Catalán, D., \& Merced, J. (2016). Utilización de las redes sociales sobre temas de salud en población universitaria de México. Revista Española de Comunicación en Salud, 7(1), 1528. http://dx.doi.org/10.20318/recs.2016.3165.

Sánchez, M., Terrón, J. L., \& Lozano, J. C. (2016). Estigmatización y usos léxicos en el tratamiento informativo del $\mathrm{VIH} / \mathrm{Sida}$ en cinco diarios mexicanos de 2012 a 2013. Comunicación y Sociedad, (1), 71-100.

Santos, J. I., Villa, J., P., García, M., A., León, G., Quezada, S., \& Tapia, R. (2003). La transición epidemiológica de las y los adolescentes en México. Salud Pública de México, 45 (Supl. 1), 140-152.

Stern, C. (1997). El embarazo en la adolescencia como problema público: una visión crítica. Salud Pública de México, 39(1), 137-143.

Stern, C. (2004). Vulnerabilidad social y embarazo adolescente en México. Papeles de Población, 10(1), 129-158.

Terrón, J. L., Ramírez, F. M., Vialás, S., \& Jacobetty, P. 
(2017). La comunicación en las revistas de salud pública de la biblioteca virtual SCIELO. Revista Española de Comunicación en Salud, 8(2), 195183. https://doi.org/10.20318/recs.2017.3999.

Torres, P., Walker, D. M., Gutiérrez, J. P., \& Bertozzi, S. M. (2006). Estrategias novedosas de prevención de embarazo e ITS/VIH/SIDA entre adolescentes escolarizados mexicanos. Salud Pública de México, 48(1), 308-316.

Uribe, J. I., Amador, G., Zacarías, X., \& Villarreal, L. (2012). Percepciones sobre el uso del condón y la sexualidad entre jóvenes. Revista Latinoamericana de Ciencias Sociales, Niñez y Juventud, 10(1), 481-494.

Villalobos, A., Campero, L., Suárez, L., Atienzo, E., Estrada, F., \& De la Vara, E. (2015). Embarazo adolescente y rezago educativo: análisis de una encuesta nacional en México. Salud Pública de México, 57(1), 135-143. 